Review Article

\title{
New Insight on Pathophysiology, Diagnosis, and Treatment of Odontogenic Maxillary Sinusitis
}

\author{
Jianhua Zhu, ${ }^{1}$ Wei Lin, ${ }^{2}$ Wenwen Yuan, ${ }^{3}$ and Lili Chen ${ }^{4}{ }^{4}$ \\ ${ }^{1}$ Graduate School of Zhejiang University, China \\ ${ }^{2}$ Department of Otorhinolaryngology, Litongde Hospital, China \\ ${ }^{3}$ Department of Stomatology, Litongde Hospital, China \\ ${ }^{4}$ Department of Stomatology, The Second Affiliated Hospital of Zhejiang University, Zhejiang, China
}

Correspondence should be addressed to Lili Chen; y216180119@zju.edu.cn

Received 11 March 2021; Revised 19 March 2021; Accepted 19 March 2021; Published 6 May 2021

Academic Editor: Songwen Tan

Copyright (c) 2021 Jianhua Zhu et al. This is an open access article distributed under the Creative Commons Attribution License, which permits unrestricted use, distribution, and reproduction in any medium, provided the original work is properly cited.

\begin{abstract}
Odontogenic maxillary sinusitis is often ignored by otolaryngologists, dentists, and imageological diagnosis doctors. Traditional treatments are often frustrating for refractory maxillary sinusitis and odontogenic maxillary sinusitis. In the last few years, new progress has been made in the diagnosis, pathophysiology, and treatment of odontogenic maxillary sinusitis. Periodontitis, polyposis, and iatrogenesis are regarded as causes of odontogenic maxillary sinusitis. Dental implant dislocation into the maxillary sinus and augmentation are the main cause of iatrogenesis compared to root canal full material. The symptoms are too similar to distinguish odontogenic maxillary sinusitis from chronic rhinosinusitis. Computed tomography is the gold standard for diagnosis, while it is difficult to rule out odontogenic maxillary sinusitis by conventional panoramic radiographs. Cone-beam computed tomography (CBCT) is currently the most broadly used to make diagnosis and differential diagnosis for maxillary sinus disease. The imaging diagnosis, clinical diagnosis, and pathological diagnosis are often not completely in accordance. Most researchers believe that odontogenic maxillary sinus infection results from the spread of apical pathogenic microbial infections: either through the local vascular system, lymphatic system, or the Haval system of the alveolar bone itself. DNA and RNA sequencing of mucosal tissues of maxillary sinus disease confirmed that the mechanisms of odontogenic maxillary sinusitis and nonodontogenic maxillary sinusitis are different. Microbial RNA sequencing of the maxillary sinus also verifies this conclusion. Clinical serum testing of chemical factors has not been widely exploited. It is unknown whether the thickening of the maxillary sinus membrane is the result of pathogen infection or inflammatory mediators. Many doctors have recommended a consensus on multidisciplinary cooperation management, but a global consensus has not yet been reached. Diagnosis methods for odontogenic maxillary sinus disease are diversified. Tissue DNA and RNA sequencing, chemical factor determination, and microbiological DNA and RNA sequencing have appeared, and the misdiagnosis rate has gradually decreased. The pathophysiology of odontogenic maxillary sinusitis and chronic rhinosinusitis is different. The molecular mechanism of the thickening of the Schneider membrane in odontogenic sinus is unknown.
\end{abstract}

\section{Background}

The first professor to propose the concept of odontogenic maxillary sinus was William H. Bauer in 1943 [1]. Over the past decades, the concept has evolved, and odontogenic maxillary sinus has become a well-recognized condition in both the dental and otolaryngology communities [2]. Periodontitis, apical periodontitis, and root canal therapy superfilling materials such as silver amalgam and dental tissue are the main motivation of odontogenic maxillary sinus infection $[3,4]$. Since the development of dental implant surgery, many documents have pointed out that iatrogenic factors such as implants and maxillary sinus lifting materials have been accounting for more and more causation [5-9]. Odontogenic maxillary sinusitis has not been documented in the ear, nose, and throat (ENT) practice guidelines in 2012, 2017, and earlier. The "European Position Paper on Rhinosinusitis and Nasal Polyps 2020" mentions the diagnosis of 


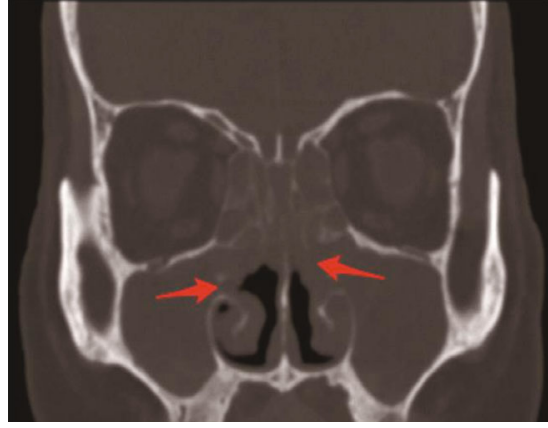

(a)



(c)

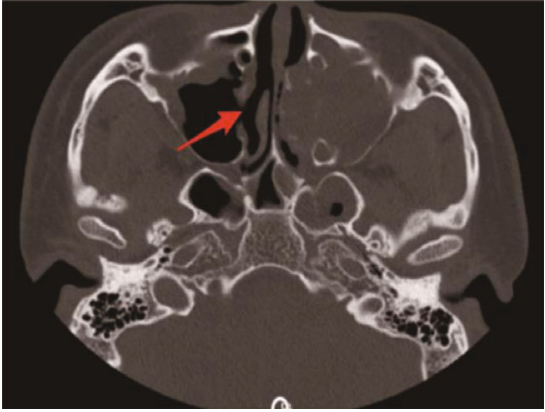

(b)

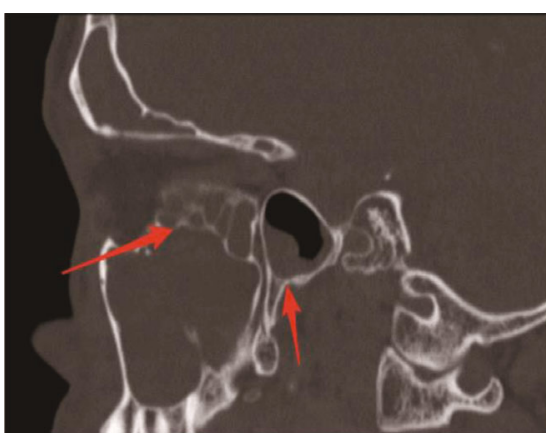

(d)

FIGURE 1: Individual with chronic maxillary sinusitis. (a) Bilateral chronic maxillary sinusitis. The arrows show that the lesions scatter over the adjacent tissues finally to the other side of the sinus. (b) The transverse image of the infected area. The arrow shows the infected area. (c) The cross-sectional view of the maxillary sinus on the right. The arrow shows that the lesion spreads to other parts of the maxillary sinuses. (d) The sagittal position of the left maxillary sinus. The arrows show that the lesion spreads to adjacent tissues and finally spreads to other maxillary sinuses.

odontogenic maxillary sinusitis in the classification of rhinosinusitis $[10,11]$. Due to the different population bases of various literature statistics, the proportion of odontogenic maxillary sinusitis diagnosis is inconsistent, about 10\%-40\% [12-14].

\section{Diagnosis}

It is inaccurate to diagnose with maxillary sinusitis depending on panoramic film alone. Computed tomography (CT) and cone-beam computed tomography (CBCT) are regarded as better options for diagnosing maxillary sinus disease which have been proven in our daily practice $[15,16]$. CBCT is currently the most commonly used method to diagnose maxillary sinusitis. However, it is not enough to diagnose maxillary sinusitis according to imaging data alone [16]. It is reported that the pathological examination results are not always in accordance with the CBCT presentation before operation [16-18]. Early differential diagnosis of maxillary sinusitis, sinus mucosa thickening, sinus cyst, maxillary sinus endometrial papillary inverted fold, and sinus mucosal bleeding is not easy $[19,20]$. Emphasis with the identification between imaging diagnosis, clinical diagnosis, or pathological diagnosis is important [16]. Dobros and Zarzecka tried to establish a diagnostic model of odontogenic maxillary sinusitis based on symptoms [4].
With the development of science, new diagnostic methods have emerged. More and more literatures which emphasize diagnosis and differential diagnosis utilizing maxillary-related cytokines and chemical factors have gradually increased [21]. Interferon- $\alpha$; interferon- $\gamma$; proinflammatory cytokines including brain-derived neurotrophic factor (BDNF), tumor necrosis factor-alpha (TNF- $\alpha$ ), and IL-3; viral-associated chemokines including IP-10 (CXCL10), ITAC (CXCL11), MCP-3 (CCL7), and MIG (CXCL9); microbial peptides, Toll-like receptors; picric acid receptors; neopterin levels; $\alpha$-defensins; and prostaglandin D2 all have been selected for laboratory detection for chronic maxillary sinusitis. However, whether it can be used for the differential diagnosis of odontogenic maxillary sinusitis from maxillary sinus needs further study [22-28], whereas cytokines and chemokines are currently detected in local tissues, and detection in serum is relatively rare. Rapoport et al. tried to compare microbial DNA probes with a conventional microbial culture to find the pathogenic microbial as well as chemosensitivity and found that DNA probes are more accurate and reliable than traditional methods [29]. However, the detection efficiency is limited and needs further expansion.

\section{Pathophysiology}

The pathological characteristics of odontogenic maxillary sinusitis and chronic sinusitis are different $[18,30]$. It is 


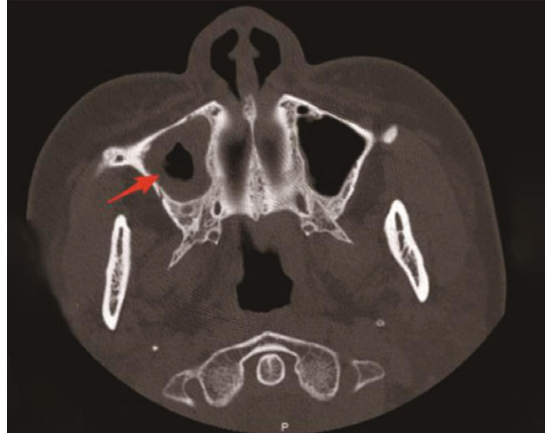

(a)



(c)

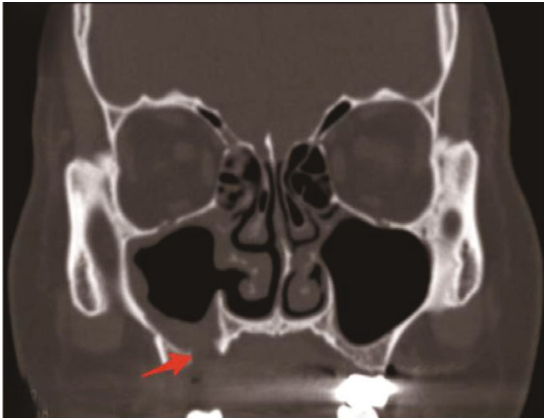

(b)

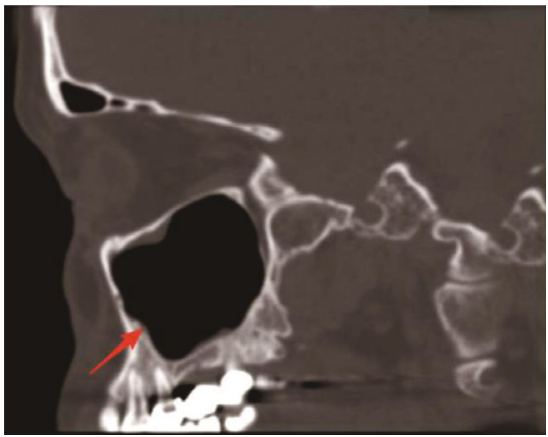

(d)

FIgURE 2: Individual with right maxillary sinusitis. (a) The transverse image of the infected area. The arrow shows that the lesions lie in the right maxillary sinus. The left sinus is normal. (b) Comparison sagittal image of left maxillary sinus in the same patient. The arrow shows communication between the oral and the maxillary sinus. (c) The coronal image of the infected area. The patient suffered from odontogenic maxillary sinusitis and had been tooth extracted through the traditional Caldwell-Luc approach. After recurrence, FESS surgery and local mucosal flap transposition were performed transorally. (d) Comparison sagittal image of right maxillary sinus in the same patient.

viewed that the pathophysiology of rhinogenic sinusitis has various reasons leading to the inflammation of the sinus ostium complex, which spreads to the tissues around the ostium, and finally to other maxillary sinus locations, blocking the ability of maxillary sinus mucosal clearance. The cascade of inflammation will lead to damage by the infiltrating cells, causing edema, fluid extravasation, mucus production, engorgement, and sinus obstruction in the process (such as Figures 1(a)-1(d)). The origin of odontogenic maxillary sinusitis is the pathogenic teeth; from the teeth, it diffuses to the maxillary sinus floor, then from the maxillary sinus floor, it spreads to the maxillary sinus ostium and finally to the tissues around the maxillary sinus ostium and other accessory sinuses. The microorganisms even scatter over the orbital and brain [20, 28, 31-35]. The odontogenic maxillary sinusitis can be used as an important basis for distinguishing odontogenic maxillary sinusitis from nonodontogenic maxillary sinusitis (such as Figures 2(a)$2(d))$.

In terms of anatomy, it is believed that the veins, arterial connections, and lymphatic system are the main way which make lesion bacteria diffuse from the periodontal to the maxillary sinus. The Haval system in the alveolar bone also acts as an important approach for evaluating the spread of infection [36]. Studies have verified this theory through autopsy, angiography, and radiographic scintigraphy $[37,38]$. There are various reasons for the thickening of the maxillary sinus mucosa. Some authors use CBCT analysis to infer that the thickening of the maxillary sinus mucosa is closely related to periodontal conditions and root apex conditions. The Schneider membranes of many patients have gradually recovered after root canal treatment $[39,40]$. It is unclear whether the thickening of the maxillary sinus mucosa and early maxillary sinus inflammation is the result of inflammatory mediators or the scatter of microorganisms to the maxillary sinus $[39,40]$. Further research and confirmation in the future are essential.

Maxillary sinusitis is the dysfunction of the host mucosal epithelium under environmental pressure to initiate an inflammatory response. The inflammatory response cascade amplifies the process along corresponding inflammatory mediators. But the complete molecular mechanism is currently unclear $[35,41]$. Interestingly, inflammatory mediators are different in various countries, even in different regions of the same country because of different susceptible microorganisms [22, 42]. The inflammatory mediators are different between odontogenic maxillary sinusitis and nonodontogenic maxillary sinusitis. The pathways involved in inflammation are also various [22, 36, 38, 43-45]. In terms of the microorganism, the identification of microbial DNA or RNA sequencing, the detection of virulence genes, and the determination of drug resistance genes are 
supplementary methods for routine culture negative results in clinical work. Microbiological gene sequencing showed that microbial communities were constant compared to normal maxillary sinus and chronic maxillary, but the species changed $[46,47]$. The microbial dominant bacteria between the odontogenic maxillary sinusitis and the chronic maxillary sinusitis are different by the microbial gene sequencing. For chronic sinus disease, anaerobic bacteria, streptococcus, Pseudomonas aeruginosa, or predominate fungi are dominated. Refractory maxillary results from microbial membrane colonization, which has yet to be confirmed by further experiments [48]. The research on proteomics and metabolomics is mainly focused on chronic maxillary, while there are limited data. Limited literature on the differential diagnosis of odontogenic maxillary has been documented $[23,45]$. Microbial DNA or RNA detection, proteomics testing, and metabolomics identification are the current and future development directions for the diagnosis and differential diagnosis of microbial infections.

\section{Treatment}

Elimination of infection source in odontogenic maxillary sinusitis is important for preventing symptom persistence. Antibiotics play a part in treating odontogenic maxillary sinusitis whenever combined with other appropriate treatments. Clindamycin and clavulanate potassium can overcome drug resistance during the treatment of chronic and odontogenic maxillary sinusitis. When maxillary sinus inflammation scatters to adjacent organs, the length of antibiotics is reported for 3 weeks to 3 months, or even longer [49]. Previous evidence showed that $50 \%$ of all odontogenic sinusitis pathogens were resistant to clindamycin, making this a poor choice for antibiotic therapy [50]. Craig et al. proposed functional endoscopic sinus surgery (FESS) as the first choice for maxillary sinus treatment prior to endodontic treatment [51]. Tsuzuki et al. proposed that extraction is the prior choice instead of FESS and dental implant [52]. Yoo et al. believe that dental endodontic treatment and medicine are given priority, and FESS is required according to the treatment results [53]. Akiyama et al. proposed that the apical surgery was performed simultaneously with FESS to achieve better results [54]. Aukstakalnis et al. provide grading odontogenic maxillary sinusitis, which is of positive significance for the selection of treatment options and cooperation among multiple departments [55]. Endodontic history and the lesions of odontogenic maxillary sinusitis are candidates for FESS [56]. Craig et al. completed a survey of experts related to odontogenic maxillary sinusitis governance in the United States and achieved consensus on the management of sinus disease. Among them, the participating dentists are relatively scarce, and the numbers of experts participating in the survey are also relatively few [57]. Some researchers proposed that dental surgery should be performed first to eliminate the source of infection, followed by sinus surgery to relieve symptoms and prevent recurrence of sinusitis [56]. The consensus needs further supplementation and improvement.

\section{Conclusion}

Odontogenic maxillary sinusitis, as a category of chronic maxillary sinus disease, is receiving more and more attention from otolaryngology and maxillofacial surgeons. Dentists and radiologists are gradually joining the management. With gradual comprehension of pathophysiology and microbiology of odontogenic maxillary sinusitis and the improvement of clinical treatment effects, more and more doctors from different countries have accepted the consensus on the management of the disease. Nevertheless, a global management consensus has not yet been signed in, and subsequent studies based on clinical evidence are warrant.

\section{Data Availability}

The data used to support the findings of this study are included within the article.

\section{Conflicts of Interest}

All authors declare that they have no conflict of interest.

\section{References}

[1] W. H. Bauer, "Maxillary sinusitis of dental origin," American Journal of Orthodontics \& Oral Surgery, vol. 29, no. 3, pp. B133-B151, 1943.

[2] H. A. Newsome and D. M. Poetker, "Odontogenic sinusitis: current concepts in diagnosis and treatment," Immunology and Allergy Clinics of North America, vol. 40, no. 2, pp. 361$369,2020$.

[3] M. N. Chapman, R. N. Nadgir, A. S. Akman et al., "Periapical lucency around the tooth: radiologic evaluation and differential diagnosis," Radiographics, vol. 33, no. 1, pp. E15-E32, 2013.

[4] K. Dobros and J. Zarzecka, "Challenges in diagnosing odontogenic lesions in maxillary sinuses," Otolaryngologia Polska, vol. 74, no. 3, pp. 12-16, 2019.

[5] M. Chiapasco, G. Felisati, A. Maccari, R. Borloni, F. Gatti, and F. Di Leo, "The management of complications following displacement of oral implants in the paranasal sinuses: a multicenter clinical report and proposed treatment protocols," International Journal of Oral and Maxillofacial Surgery, vol. 38, no. 12, pp. 1273-1278, 2009.

[6] J. R. Lechien, R. Lamartine Monteiro, S. Kampouridis, R. Javadian, and M. Horoi, "Unilateral chronic maxillary rhinosinusitis after bone maxillary graft for dental implant placement: a case report," Clinical Case Reports, vol. 8, no. 9, pp. 1827-1830, 2020.

[7] Y. Manor, Y. Anavi, R. Gershonovitch, A. Lorean, and E. Mijiritsky, "Complications and management of implants migrated into the maxillary sinus," The International Journal of Periodontics \& Restorative Dentistry, vol. 38, no. 6, pp. e112-e118, 2018.

[8] P. Galindo-Moreno, M. Padial-Molina, G. Avila, H. F. Rios, P. Hernandez-Cortes, and H. L. Wang, "Complications associated with implant migration into the maxillary sinus cavity," Clinical Oral Implants Research, vol. 23, no. 10, pp. 1152 1160, 2012. 
[9] A. Safadi, O. J. Ungar, I. Oz, I. Koren, A. Abergel, and S. Kleinman, "Endoscopic sinus surgery for dental implant displacement into the maxillary sinus-a retrospective clinical study," International Journal of Oral and Maxillofacial Surgery, vol. 49, no. 7, pp. 966-972, 2020.

[10] W. J. Fokkens, V. J. Lund, C. Hopkins et al., "European position paper on rhinosinusitis and nasal polyps 2020," Rhinology, vol. 58, Suppl S29, 2020.

[11] W. J. Fokkens, "EPOS2020: a major step forward," Rhinology, vol. 58, no. 1, p. 1, 2020.

[12] S. Cartwright and C. Hopkins, "Odontogenic sinusitis an underappreciated diagnosis: our experience," Clinical Otolaryngology, vol. 41, no. 3, pp. 284-285, 2016.

[13] Z. Turfe, A. Ahmad, E. I. Peterson, and J. R. Craig, "Odontogenic sinusitis is a common cause of unilateral sinus disease with maxillary sinus opacification," International Forum of Allergy \& Rhinology, vol. 9, no. 12, pp. 1515-1520, 2019.

[14] A. Wuokko-Landen, K. Blomgren, A. Suomalainen, and H. Valimaa, "Odontogenic causes complicating the chronic rhinosinusitis diagnosis," Clinical Oral Investigations, vol. 25, no. 3, pp. 947-955, 2021.

[15] A. D. Miller, "Respiratory muscle control during vomiting," Canadian Journal of Physiology and Pharmacology, vol. 68, no. 2, pp. 237-241, 1990.

[16] Z. Huang, H. Xu, N. Xiao et al., "Predictive significance of radiographic density of sinus opacity and bone thickness in unilateral maxillary sinus mycetoma," ORL: Journal for Otorhinolaryngology and Its Related Specialties, vol. 81, no. 2-3, pp. 111-120, 2019.

[17] I. Oreski, T. Greguric, P. Gulin, N. Prica Oreski, D. Brajdic, and D. Vagic, "Differences in self-reported symptoms in patients with chronic odontogenic and non-odontogenic rhinosinusitis," American Journal of Otolaryngology, vol. 41, no. 2, p. 102388, 2020.

[18] A. Raman, P. Papagiannopoulos, H. N. Kuhar, P. Gattuso, P. S. Batra, and B. A. Tajudeen, "Histopathologic features of chronic sinusitis precipitated by odontogenic infection," American Journal of Rhinology \& Allergy, vol. 33, no. 2, pp. 113-120, 2019.

[19] H. Wang, Z. Tan, M. You, and W. J. Liu, "Imaging classification diagnosis and maxillary sinus floor augmentation of maxillary sinus cystic lesions," Hua Xi Kou Qiang Yi Xue Za Zhi, vol. 37, no. 5, pp. 457-462, 2019.

[20] M. Sakir and S. Ercalik Yalcinkaya, "Associations between periapical health of maxillary molars and mucosal thickening of maxillary sinuses in cone-beam computed tomographic images: a retrospective study," Journal of Endodontia, vol. 46, no. 3, pp. 397-403, 2020.

[21] K. Scheckenbach and M. Wagenmann, "Cytokine patterns and endotypes in acute and chronic rhinosinusitis," Current Allergy and Asthma Reports, vol. 16, no. 1, p. 3, 2016.

[22] X. Wang, N. Zhang, M. Bo et al., "Diversity of TH cytokine profiles in patients with chronic rhinosinusitis: a multicenter study in Europe, Asia, and Oceania," The Journal of Allergy and Clinical Immunology, vol. 138, no. 5, pp. 1344-1353, 2016.

[23] S. M. Daines, Y. Wang, and R. R. Orlandi, "Periostin and osteopontin are overexpressed in chronically inflamed sinuses," International Forum of Allergy \& Rhinology, vol. 1, no. 2, pp. 101-105, 2011.

[24] Y. Sun, B. Zhou, C. Wang et al., "Biofilm formation and tolllike receptor 2, toll-like receptor 4, and NF-kappaB expression in sinus tissues of patients with chronic rhinosinusitis," American Journal of Rhinology \& Allergy, vol. 26, no. 2, pp. 104-109, 2012.

[25] M. Tizzano, B. D. Gulbransen, A. Vandenbeuch et al., "Nasal chemosensory cells use bitter taste signaling to detect irritants and bacterial signals," Proceedings of the National Academy of Sciences of the United States of America, vol. 107, no. 7, pp. 3210-3215, 2010.

[26] R. J. Lee, G. Xiong, J. M. Kofonow et al., “T2R38 taste receptor polymorphisms underlie susceptibility to upper respiratory infection," The Journal of Clinical Investigation, vol. 122, no. 11, pp. 4145-4159, 2012.

[27] X. Feng, M. K. Ramsden, J. Negri et al., "Eosinophil production of prostaglandin $\mathrm{D}_{2}$ in patients with aspirin-exacerbated respiratory disease," Journal of Allergy and Clinical Immunology, vol. 138, no. 4, pp. 1089-1097.e3, 2016.

[28] T. G. Stead, A. Retana, J. Houck, B. C. Sleigh, and L. Ganti, "Preseptal and postseptal orbital cellulitis of odontogenic origin," Cureus, vol. 11, no. 7, 2019.

[29] S. K. Rapoport, A. J. Smith, M. Bergman, K. A. Scriven, I. Brook, and S. K. Mikula, "Determining the utility of standard hospital microbiology testing: comparing standard microbiology cultures with DNA sequence analysis in patients with chronic sinusitis," World Journal of Otorhinolaryngology Head and Neck Surgery, vol. 5, no. 2, pp. 82-87, 2019.

[30] N. R. London Jr., I. Lina, and M. Ramanathan Jr., "Aeroallergens, air pollutants, and chronic rhinitis and rhinosinusitis," World Journal of Otorhinolaryngology - Head and Neck Surgery, vol. 4, no. 3, pp. 209-215, 2018.

[31] A. M. Saibene, G. C. Pipolo, P. Lozza et al., "Redefining boundaries in odontogenic sinusitis: a retrospective evaluation of extramaxillary involvement in 315 patients," International Forum of Allergy \& Rhinology, vol. 4, no. 12, pp. 1020-1023, 2014.

[32] K. V. Arunkumar, "Orbital infection threatening blindness due to carious primary molars: an interesting case report," Journal of Maxillofacial and Oral Surgery, vol. 15, no. 1, pp. 72-75, 2016.

[33] A. Safadi, S. Kleinman, I. Oz et al., "Questioning the justification of frontal sinusotomy for odontogenic sinusitis," Journal of Oral and Maxillofacial Surgery, vol. 78, no. 5, pp. 762-770, 2020.

[34] A. Laulajainen-Hongisto, L. Lempinen, E. Farkkila et al., "Intracranial abscesses over the last four decades; changes in aetiology, diagnostics, treatment and outcome," Infectious Diseases, vol. 48, no. 4, pp. 310-316, 2015.

[35] M. Boruk, C. Railwah, A. Lora et al., "Elevated S100A9 expression in chronic rhinosinusitis coincides with elevated MMP production and proliferation in vitro," Scientific Reports, vol. 10, no. 1, p. 16350, 2020.

[36] I. G. Pereira, P. Vaz, R. F. Almeida, A. C. Braga, and A. Felino, "IRAK4 gene polymorphism and odontogenic maxillary sinusitis," Clinical Oral Investigations, vol. 19, no. 8, pp. 1815-1824, 2015.

[37] C. Glaser, M. Pruckmayer, A. Staudenherz, M. Rasse, S. Lang, and T. Leitha, "Utility of technetium-99m-sestamibi to assess osseous tumor spread," Journal of Nuclear Medicine, vol. 37, no. 9, pp. 1526-1528, 1996.

[38] A. A. Haider, M. J. Marino, W. C. Yao, M. J. Citardi, and A. U. Luong, "The potential of high-throughput DNA sequencing of the paranasal sinus microbiome in diagnosing odontogenic 
sinusitis," Otolaryngology and Head and Neck Surgery, vol. 161, no. 6, pp. 1043-1047, 2019.

[39] U. Aksoy and K. Orhan, "Association between odontogenic conditions and maxillary sinus mucosal thickening: a retrospective CBCT study," Clinical Oral Investigations, vol. 23, no. 1, pp. 123-131, 2019.

[40] T. Van Den Munckhof, S. Patel, G. Koller, E. Berkhout, F. Mannocci, and F. Foschi, "Schneiderian membrane thickness variation following endodontic procedures: a retrospective cone beam computed tomography study," BMC Oral Health, vol. 20, no. 1, p. 133, 2020.

[41] Y. J. Tsai, C. Y. Hao, C. L. Chen, P. H. Wu, and W. B. Wu, "Expression of long pentraxin 3 in human nasal mucosa fibroblasts, tissues, and secretions of chronic rhinosinusitis without nasal polyps," Journal of Molecular Medicine (Berlin, Germany), vol. 98, no. 5, pp. 673-689, 2020.

[42] D. K. Kim, K. M. Eun, M. K. Kim et al., "Comparison between signature cytokines of nasal tissues in subtypes of chronic rhinosinusitis," Allergy, Asthma \& Immunology Research, vol. 11, no. 2, pp. 201-211, 2019.

[43] T. Van Zele, S. Claeys, P. Gevaert et al., "Differentiation of chronic sinus diseases by measurement of inflammatory mediators," Allergy, vol. 61, no. 11, pp. 1280-1289, 2006.

[44] V. K. Anand, A. Kacker, A. F. Orjuela, C. Huang, C. Manarey, and J. Xiang, "Inflammatory pathway gene expression in chronic rhinosinusitis," American Journal of Rhinology, vol. 20, no. 4, pp. 471-476, 2018.

[45] C. Jardeleza, D. Jones, L. Baker et al., "Gene expression differences in nitric oxide and reactive oxygen species regulation point to an altered innate immune response in chronic rhinosinusitis," International Forum of Allergy \& Rhinology, vol. 3, no. 3, pp. 193-198, 2013.

[46] V. R. Ramakrishnan, L. J. Hauser, L. M. Feazel, D. Ir, C. E. Robertson, and D. N. Frank, "Sinus microbiota varies among chronic rhinosinusitis phenotypes and predicts surgical outcome," Journal of Allergy and Clinical Immunology, vol. 136, no. 2, pp. 334-342.e1, 2015.

[47] V. R. Ramakrishnan, L. M. Feazel, L. J. Abrass, and D. N. Frank, "Prevalence and abundance of Staphylococcus aureus in the middle meatus of patients with chronic rhinosinusitis, nasal polyps, and asthma," International Forum of Allergy \& Rhinology, vol. 3, no. 4, pp. 267-271, 2013.

[48] J. H. Fastenberg, W. D. Hsueh, A. Mustafa, N. A. Akbar, and W. M. Abuzeid, "Biofilms in chronic rhinosinusitis: pathophysiology and therapeutic strategies," World Journal of Otorhinolaryngology - Head and Neck Surgery, vol. 2, no. 4, pp. 219-229, 2016.

[49] B. P. Erickson and W. W. Lee, "Orbital cellulitis and subperiosteal abscess: a 5-year outcomes analysis," Orbit, vol. 34, no. 3, pp. 115-120, 2015.

[50] S. Puglisi, S. Privitera, L. Maiolino et al., "Bacteriological findings and antimicrobial resistance in odontogenic and nonodontogenic chronic maxillary sinusitis," Journal of Medical Microbiology, vol. 60, no. 9, pp. 1353-1359, 2011.

[51] J. R. Craig, C. I. McHugh, Z. H. Griggs, and E. I. Peterson, "Optimal timing of endoscopic sinus surgery for odontogenic sinusitis," Laryngoscope, vol. 129, no. 9, pp. 1976-1983, 2019.

[52] K. Tsuzuki, K. Kuroda, K. Hashimoto et al., "Odontogenic chronic rhinosinusitis patients undergoing tooth extraction: oral surgeon and otolaryngologist viewpoints and appropriate management," The Journal of Laryngology and Otology, vol. 134, no. 3, pp. 241-246, 2020.

[53] B. J. Yoo, S. M. Jung, H. N. Lee, H. G. Kim, J. H. Chung, and J. H. Jeong, "Treatment strategy for odontogenic sinusitis," American Journal of Rhinology \& Allergy, vol. 35, no. 2, pp. 206-212, 2021.

[54] K. Akiyama, Y. Nakai, Y. Samukawa, M. Miyake, and H. Hoshikawa, "Assessment of simultaneous surgery for odontogenic sinusitis," The Journal of Craniofacial Surgery, vol. 30, no. 1, pp. 239-243, 2019.

[55] R. Aukstakalnis, R. Simonaviciute, and R. Simuntis, "Treatment options for odontogenic maxillary sinusitis: a review," Stomatologija, vol. 20, no. 1, pp. 22-26, 2018.

[56] J. L. Mattos, B. J. Ferguson, and S. Lee, "Predictive factors in patients undergoing endoscopic sinus surgery for odontogenic sinusitis," International Forum of Allergy \& Rhinology, vol. 6, no. 7, pp. 697-700, 2016.

[57] J. R. Craig, R. W. Tataryn, T. L. Aghaloo et al., "Management of odontogenic sinusitis: multidisciplinary consensus statement," International Forum of Allergy \& Rhinology, vol. 10, no. 7, pp. 901-912, 2020. 